Artículo de investigación

\title{
Evaluación genética para características de peso en ganado Brahman comercial
}

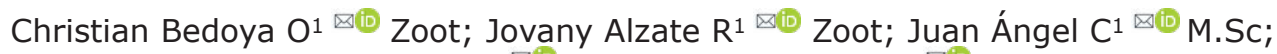 \\ Carlos Escobar R ${ }^{2}$ M.Sc; Samir Calvo C ${ }^{1 *} \bowtie \mathbb{0}$ Ph.D.
}

${ }^{1}$ Universidad Católica de Oriente, Facultad de Ciencias Agropecuarias, Grupo de investigación en agronomía y zootecnia GIAZ, Rionegro-Antioquia.

2Universidad de Antioquia, Facultad de Ciencias Agrarias, Grupo de Investigación en Ciencias Agrarias-GRICA, Medellín-Antioquia.

*Correspondencia: samirmejoramiento@gmail.com

Received: May 2018; Accepted: November 2018; Published: May 2019.

\section{RESUMEN}

Objetivo. Evaluar genéticamente las características de peso al nacimiento y peso al destete en una población de ganado Brahman comercial. Materiales y métodos. Se analizaron 1.015 registros de peso al nacimiento (PN) y peso al destete ajustado a los 270 días (PA270) de crías entre los años 2002 y 2014 pertenecientes a la hacienda La Envidia, ubicada en el municipio de Planeta Rica, Córdoba. Se utilizó un modelo animal bi-carácter que incluyó efectos fijos de sexo, mes, año y edad al destete, efecto genético aditivo y el efecto materno, estimando los parámetros genéticos con el software MTDF-REML. Resultados. Se encontró un promedio para PN de $29.62 \pm 3.13 \mathrm{~kg}$ y coeficiente de variación (CV) del $10.6 \%$. La media para PA270 fue de $216.713 \pm 26.97 \mathrm{~kg}$ y CV $12.5 \%$. Los estimados de heredabilidad directa $\left(\mathrm{h}^{2} \mathrm{~d}\right)$ fueron de $0.44 \pm 0.14$ y $0.39 \pm 0.12$ para PN y PA270, respectivamente. La heredabilidad materna ( $\mathrm{h}^{2} \mathrm{~m}$ ) fue de $0.09 \pm 0.092$ y $0.42 \pm 0.087$ para PN y PA270, respectivamente. La correlación genética y fenotípica estimada entre las características evaluadas fue de 0.11 y 0.13 , respectivamente; con progreso genético por año para PN de $-2.02 \mathrm{~kg}$ y 7.81 $\mathrm{kg}$ para PA270. Conclusiones. Las estimaciones de heredabilidad indican la existencia de variabilidad genética aditiva directa, evidenciando la oportunidad de ganancia genética por medio de selección. Sin embargo, las características de peso al nacer y peso del destete presentan gran influencia del ambiente, por lo cual se debe poner especial atención al manejo y la nutrición de las hembras durante la gestación y de los terneros desde el nacimiento hasta el destete.

Palabras clave: Correlación genética, ganado de carne, heredabilidad, modelo animal, progreso genético (Fuente: CAB).

\begin{abstract}
Objective. Evaluate genetically the traits of birth weight and weaning weight in commercial Brahman cattle. Materials and methods. A total of 1.015 records of birth weight (BWT) and weaning weight adjusted to 270 days (WW270), were evaluated. Data correspond to the offspring born between 2002 and 2014 belonging to "La Envidia" farm, located in the municipality of Planeta Rica, Córdoba. A bi-character animal model that included, additive genetic effect, maternal effect and sex, month, year and age at weaning as fixed effects, was used. Genetic parameters were estimated using the MTDF-REML software. Results. The average and coefficient of variation (CV) of BWT was $29.62 \pm 3.13 \mathrm{~kg}$ and $10.59 \%$, respectively. The mean and CV for WW270 was $216.71 \pm 26.97 \mathrm{~kg}$ and $12.45 \%$, respectively. The estimates of direct heritability were $0.44 \pm 0.143$ and $0.39 \pm 0.12$ for BWT and WW270, respectively. Maternal heritability was $0.09 \pm 0.092$ and $0.42 \pm 0.087$ for BWT and WW270, respectively. The estimated genetic and phenotypic correlation between the evaluated characteristics was 0.11 and 0.13 , respectively; with genetic progress per year of $-2.02 \mathrm{~kg}$ for BWT and $7.81 \mathrm{~kg}$ for WW270. Conclusion. The estimations of heritability indicate the existence of direct additive genetic variability, evidencing the opportunity of genetic gain through selection. However, environment have a great influence on birth weight and weaning weight traits, which is why special attention should be paid to the handling and nutrition of females during pregnancy and of calves from birth to weaning.
\end{abstract}

Keywords: Animal model, beef cattle, genetic correlation, genetic progress, heritability, (Source: CAB).

Como citar (Vancouver)

Bedoya OC, Alzate RJ, Ángel CJ, Escobar RC, Calvo CS. Evaluación genética para características de peso en ganado Brahman comercial. Rev MVZ Cordoba. 2019; 24(2):7225-7230. DOI: https://doi.org/10.21897/rmvz.1662

(C)EI (los) autor (es), Revista MVZ Córdoba 2019. Este artículo se distribuye bajo los términos de la licencia internacional Creative Commons Attribution 4.0 (https://creativecommons.org/licenses/by-sa/4.0/), que permite el uso sin restricciones, la distribución y la reproducción en cualquier medio, siempre que se otorgue el crédito apropiado al autor o autores originales y la fuente. 


\section{INTRODUCCIÓN}

Según las perspectivas mundiales de la $\mathrm{FAO}$, se prevé que la producción mundial de carne en 2018 aumente a 336 millones de toneladas en equivalente de peso en canal; es decir, un 1,7 por ciento ( 6 millones de toneladas) más que en 2017 (1). En Colombia la producción de carne bovina fue de aproximadamente 905 mil toneladas en canal en el año 2017; 5 mil toneladas menos que en el año anterior (2). Teniendo en cuenta esto, es importante mencionar que Colombia es un país que presenta condiciones agroecológicas tropicales favorables para la producción de forraje durante todo el año; lo que se considera una condición positiva en la producción de carne bovina (3). Específicamente el Valle del río Sinú es una zona ganadera por excelencia y se caracteriza por tener una temperatura promedio de $27^{\circ} \mathrm{C}$, humedad relativa de $83 \%$ y zonificación agroecológica de bosque seco tropical (4). La mayoría de centros productivos ubicados en estas zonas, se dedican a la cría, levante y ceba de animales comerciales para carne, en su mayoría poblaciones de ganado Brahman producidos a partir de apareamientos no direccionados, ni con criterios de selección (3).

Si se quiere hacer de la ganadería de carne un negocio más rentable, es necesaria la toma de registros de pesajes al nacimiento y al destete como estrategia para mejorar estos parámetros de crecimiento de generación en generación. Estas características de peso están controladas por factores genéticos aditivos del padre y de la madre, factores genéticos aditivos maternos (producción de leche) y efecto ambiental directo, como manejo nutricional etc. $(5,6,7)$. Un excelente programa de evaluación genética, selección, apareamiento y manejo de estas características de peso, hacen que las poblaciones seleccionadas generen progenies mejoradas, más precoces y más rentables para las ganaderías de carne comerciales (3).

El objetivo de esta investigación fue evaluar genéticamente las características de peso al nacimiento y peso al destete ajustado a los 270 días en un hato de Brahman comercial en el valle del Sinú-Colombia.

\section{MATERIALES Y MÉTODOS}

Animales y condiciones agroecológicas. Se realizó la colecta de información fenotípica y genealógica de madres, padres y crías de una vacada no seleccionada de Brahman comercial durante el año 2002 hasta el año 2014. Los registros fueron tomados de la base de datos de la hacienda La Envidia ubicada en el valle del Sinú, departamento de Córdoba, Colombia. La hacienda se ubica a una altura de $18 \mathrm{msnm}$, temperatura promedio de $35^{\circ} \mathrm{C}$ y precipitación media anual de $1.156 \mathrm{~mm}$. Se encuentra en zona de vida bosque seco tropical (bs-T), con suelos clasificados en el orden molisol, ricos en materia orgánica y con pendientes inferiores al 5\% (8).

Alimentación y manejo. La finca es una ganadería de cría con hembras bovinas de la raza Brahman comercial, manejada bajo un sistema de apareamiento por monta natural e inseminación artificial a partir del año 2010. Los animales se manejaban bajo un sistema de pastoreo rotacional con potreros entre 4 y 6 hectáreas, establecidos con Braquipará (Brachiaria plantaginea), Pangola (Digitaria decumbens) y Angleton (Dichantium aristatumbenth), con 45-90 días de descanso dependiendo de la época del año; adicionalmente se les suministraba sal proteinada, grasa de sobrepaso y agua a voluntad.

Datos genealógicos y fenotípicos. Para la evaluación genética se recolectó la siguiente información: Identificación del animal, identificación del padre y de la madre, fecha de nacimiento de la cría, peso al nacimiento y sexo de la cría, peso y edad al destete. El número de registros para las características de la investigación fue de 1,015 datos en total. Los datos genealógicos fueron depurados usando el software especializado pedigree viewer (9) y los datos fenotípicos usando el programa R-project versión 3.5.1 (10).

A partir de los datos recolectados en finca, fue calculado el peso al destete ajustado a los 270 días usando la siguiente fórmula:

$$
P A 270=\left(\frac{\text { PESO ACTUAL }- \text { PN }}{\text { EDAD AL DESTETE EN DÍAS }}\right) * 270+P N
$$

Dónde: PA270 es peso ajustado a los 270 días y PN es el peso al nacimiento.

Modelo estadístico. La evaluación genética se realizó usando el siguiente modelo animal bi-carácter:

$$
\left[\begin{array}{l}
Y_{1} \\
Y_{2}
\end{array}\right]=\left[\begin{array}{cc}
X_{1} & 0 \\
0 & X_{2}
\end{array}\right]\left[\begin{array}{l}
\beta_{1} \\
\beta_{2}
\end{array}\right]+\left[\begin{array}{cc}
Z_{1} & 0 \\
0 & Z_{2}
\end{array}\right]\left[\begin{array}{l}
a_{1} \\
a_{2}
\end{array}\right]+\left[\begin{array}{cc}
W_{1} & 0 \\
0 & W_{2}
\end{array}\right]\left[\begin{array}{l}
m_{1} \\
m_{2}
\end{array}\right]+\left[\begin{array}{l}
e_{1} \\
e_{2}
\end{array}\right]
$$

Donde;

$Y_{1}, Y_{2}$ : Vector de observaciones ( $Y_{1}=\mathrm{PN}$ y $Y_{2:}$ PA270).

$X_{1}, X_{2}$ : Matrices de incidencia que relaciona las observaciones a los efectos fijos (sexo, año de nacimiento, mes de nacimiento, año de destete, mes de destete).

$B_{1}, \beta_{2}$ : Soluciones para los efectos fijos de peso al nacimiento y peso al destete.

$Z_{1}, Z_{2}$. Matriz de incidencia que relaciona las observaciones de efectos genéticos aditivos directos.

$a_{1}, a_{2}$ : Soluciones para los efectos aleatorios directos (valor de cría esperado).

$W_{1}, W_{2}$ : Matriz de incidencia que relaciona el efecto aleatorio materno.

$m_{1}, m_{2}$ : Soluciones para los efectos maternos.

$e_{1}, e_{2}$ : Error estadístico.

Análisis de correlación. Para estimar la correlación fenotípica entre las características de PN y PA270, se utilizó la ecuación planteada por Falconer y Mackay (11), descrita a continuación:

$r p_{1,2}=\left(r g_{1,2} * \sqrt{h_{1}^{2}} * \sqrt{h_{2}^{2}}\right)+\left(r a_{1,2} * \sqrt{1-h_{1}^{2}} * \sqrt{1-h_{2}^{2}}\right)$

Donde:

$r p_{1,2}$ : Correlación fenotípica entre las características 1 y 2

$r g_{1,2}$ : Correlación genética entre las características 1 y 2

$\mathrm{ra}_{1,2}$ : Correlación ambiental entre las características 1 y 2

$h^{2}{ }_{1 \text { : }}$. Heredabilidad de la característica 1 
$h^{2}$. Heredabilidad de la característica 2

La estimación de componentes de varianza y los parámetros genéticos se realizó por medio del método de máxima verosimilitud restricta (REML) utilizando el software MTDF-REML (12).

El progreso genético fue estimado por medio de la siguiente fórmula (13):

$\Delta G / a \tilde{\mathrm{n}} o=\frac{h^{2} * S}{I G}$

Donde:

$\Delta \mathrm{G}$ : Progreso genético

$\mathrm{h}^{2}$ : Heredabilidad de la característica

S: Diferencial de selección

IG: Intervalo generacional, visto como el promedio ponderado de machos y hembras de la población evaluada.

\section{RESULTADOS}

En la matriz de parentesco se encontraron 1501 animales distribuidos en tres generaciones ( 83 padres, 314 madres y 795 individuos en la progenie); 66 familias paternas y 10 familias maternas. El coeficiente de endogamia promedio fue de 0.0 con 0 animales consanguíneos en la genealogía.

El promedio para PN fue de $29.62 \pm 3.13 \mathrm{Kg}$ con un CV de $10.6 \%$ y valores mínimos de 20 y máximo de $40 \mathrm{~kg}$. La media para PA270 fue $216,71 \pm 26.97 \mathrm{Kg}$ y CV de $12.5 \%$; con un valor mínimo y máximo de peso de 109.6 y $260.64 \mathrm{~kg}$, respectivamente. En la tabla 1 se puede observar la estadística descriptiva para las características de peso al nacimiento y peso al destete en la población de Brahman evaluada.

Tabla 1. Estadística descriptiva para las características del peso al nacimiento y peso al destete en ganado Brahman comercial.

\begin{tabular}{|c|c|c|c|c|c|}
\hline Características & $\mathbf{N}$ & $X \pm$ D.E & C.V & Min & Max \\
\hline PN (kg) & 1015 & $\begin{array}{l}29.62 \\
\pm 3.13\end{array}$ & 10.6 & 20 & 40 \\
\hline $\mathrm{PA} 270(\mathrm{~kg})$ & 1015 & $\begin{array}{l}216.71 \\
\pm 26.97\end{array}$ & 12.5 & 109.6 & 260.6 \\
\hline
\end{tabular}

$\mathrm{N}=$ Número de muestras, $\mathrm{X}=$ Media, $\mathrm{D} . \mathrm{E}=$ Desviación estándar,

$\mathrm{C} . \mathrm{V}=$ Coeficiente de variación, Max= Peso máximo, Min= Peso mínimo.

Los componentes de varianza, parámetros genéticos y fenotípicos para las características PN y PD encontrados en ganado Brahman comercial se pueden observar en la tabla 2 y figura 1 . La heredabilidad directa y materna para peso al nacimiento fue de $0.23 \pm 0.086$ y $0.05 \pm 0.05$, respectivamente y para PA270 fue $0.39 \pm 0.127$ y $0.39 \pm 0.091$, respectivamente. La correlación genética (0.11) y fenotípica (0.13) entre las características de PN y PA270 en la población de Brahman comercial evaluada, fueron positivas y muy bajas.
El progreso genético por año para PN y PA270 fue de -2.02 kg y $7.81 \mathrm{~kg}$, respectivamente. En la tabla 3 se pueden observar los 10 mejores animales y las 10 mejores madres para PN y PA270 con respecto a la evaluación genética realizada por el presente estudio para Brahman comercial. El mejor animal para peso al nacimiento fue la hembra "1022" con una DEP de $-3.12 \mathrm{~kg}$ y para peso al destete la hembra " 48 " con una DEP de $38.34 \mathrm{~kg}$. La mejor madre para peso al nacimiento fue la "1020" con una DEP materna de $-1.48 \mathrm{~kg}$ y para peso al destete la mejor madre fue el animal "326" con una DEP materna de $23.35 \mathrm{~kg}$.

Tabla 2. Componentes de varianza, parámetros genéticos y fenotípicos para las características del peso al nacimiento y peso al destete en ganado Brahman comercial.

\begin{tabular}{|c|c|c|c|c|c|c|c|}
\hline Características & $\sigma^{2} \mathbf{a}$ & $\sigma^{2} \mathbf{m}$ & $\sigma^{2} e$ & $\sigma^{2} p$ & $\begin{array}{l}h^{2} \mathbf{d} \\
\pm E E\end{array}$ & $\begin{array}{l}h^{2} m \\
\pm E E\end{array}$ & $\begin{array}{l}\Delta \mathbf{G} / \\
\text { año }\end{array}$ \\
\hline PN & 4.04 & 0.81 & 5.4 & 9.21 & $\begin{array}{c}0.44 \\
\pm 0.14\end{array}$ & $\begin{array}{c}0.09 \\
\pm 0.092\end{array}$ & -2.02 \\
\hline PA270 & 348.9 & 376.72 & 224.27 & 657.79 & $\begin{array}{c}0.39 \\
\pm 0.12\end{array}$ & $\begin{array}{c}0.42 \\
\pm 0.087\end{array}$ & 7.81 \\
\hline
\end{tabular}

$\sigma^{2} \mathrm{a}$ : Varianza aditiva. $\sigma^{2} \mathrm{~m}$ : Varianza materna. $\sigma^{2} \mathrm{e}$ : Varianza ambiental. $\sigma^{2} \mathrm{p}$ : Varianza fenotípica. $\mathrm{h}^{2} \mathrm{~d} \pm \mathrm{EE}$ : Heredabilidad directa \pm error estándar. $\mathrm{h}^{2} \mathrm{~m} \pm \mathrm{EE}$ : Heredabilidad materna \pm error estándar. $\Delta \mathrm{G} / \mathrm{año}$ : Progreso genético por año.

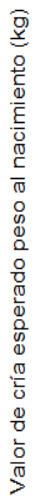

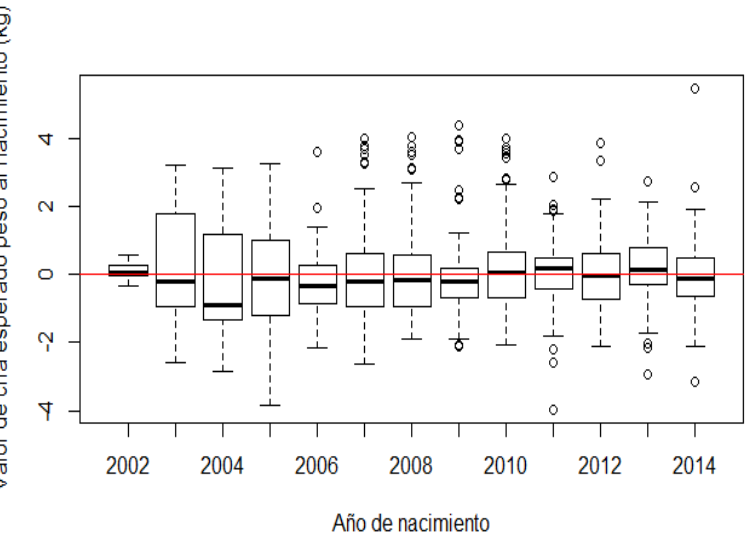

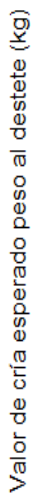

Año de nacimiento

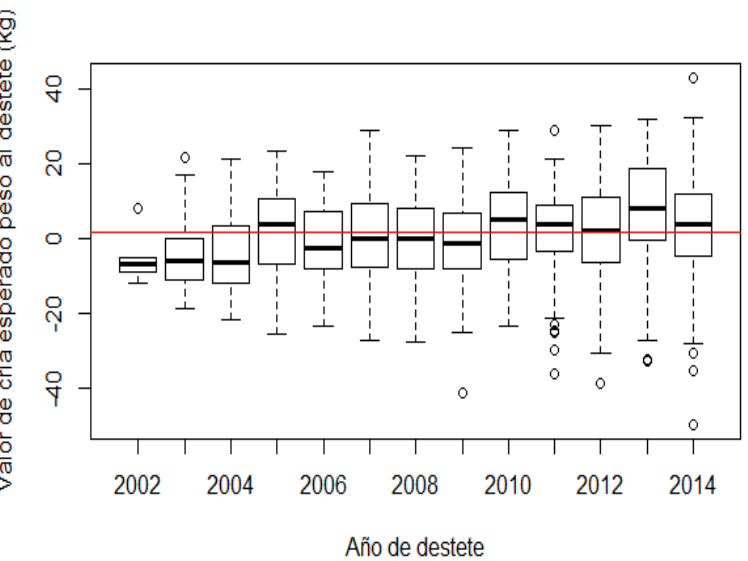

Figura 1. Comportamiento de los valores genéticos para peso al nacimiento y peso al destete por año en una población de ganado Brahman comercial no seleccionada. 
Tabla 3. Ranking de animales con los mejores valores genéticos directos y maternos para las características de peso al nacimiento (PN) y peso al destete (PD) en una población de Brahman comercial.

\begin{tabular}{|c|c|c|c|c|c|c|c|c|c|}
\hline \multicolumn{6}{|c|}{ Peso al Nacimiento } & \multicolumn{4}{|c|}{ Peso al Destete } \\
\hline ID & Sexo & DEPdir & ID Madres & DEPmat & ID & Sexo & DEPdir & ID Madres & DEPmat \\
\hline 1022 & $\mathrm{H}$ & -3.12 & 1020 & -1.48 & 48 & $\mathrm{H}$ & 38.34 & 326 & 23.35 \\
\hline 399 & $\mathrm{H}$ & -2.68 & 399 & -1.27 & 1184 & $\mathrm{H}$ & 32.19 & 554 & 18.12 \\
\hline 14 & M & -2.68 & 91 & -1.27 & 1194 & $M$ & 31.04 & 510 & 17.77 \\
\hline 91 & $\mathrm{H}$ & -2.59 & 931 & -1.23 & 1401 & $\mathrm{H}$ & 29.08 & 168 & 16.45 \\
\hline 933 & $\mathrm{H}$ & -2.35 & 1332 & -1.11 & 69 & $\mathrm{H}$ & 28.62 & 356 & 15.91 \\
\hline 1335 & $\mathrm{H}$ & -2.14 & 407 & -1.02 & 1424 & $\mathrm{H}$ & 27.51 & 655 & 15.75 \\
\hline 342 & $M$ & -2.14 & 1417 & -1.01 & 1055 & $M$ & 27.31 & 129 & 15.71 \\
\hline 407 & $\mathrm{H}$ & -2.06 & 184 & -0.98 & 73 & $\mathrm{H}$ & 27.30 & 213 & 15.70 \\
\hline 883 & $M$ & -1.95 & 1054 & -0.92 & 1144 & $\mathrm{H}$ & 26.18 & 365 & 15.58 \\
\hline 1420 & $\mathrm{H}$ & -1.94 & 375 & -0.92 & 1091 & $\mathrm{H}$ & 25.40 & 368 & 15.19 \\
\hline
\end{tabular}

ID: Identificación del animal. H: Hembra. M: Macho. DEPdir: Diferencia esperada de progenie directa. DEPmat: Diferencia esperada de progenie materna.

\section{DISCUSIÓN}

En Colombia con ganado Brahman, otros autores han encontrado pesos al nacimiento bajo condiciones de pastoreo promedios de $35 \pm 3 \mathrm{~kg}$ y $34.88 \pm 3.9 \mathrm{~kg}$, respectivamente $(3,14)$. Esto demuestra que hay diferencias principalmente en el manejo de los hatos comerciales en el trópico colombiano. Es posible que el peso promedio de la población evaluada en el presente estudio haya sido menor, dado que las condiciones de manejo y alimentación en cada finca comercial son diferentes y este centro de producción específicamente tiene un manejo extensivo, sin suplementación y cargas de animales muy altas.

Los pesos al nacimiento obtenidos en este trabajo son similares a los obtenidos por otros autores en Brasi y México, donde evaluaron ganado Nelore y Brahman comercial y encontraron medias de $32.2 \pm 1.8 \mathrm{~kg}$ y $32.30 \pm 3.80 \mathrm{~kg}$, respectivamente $(15,16)$. En Colombia también evaluaron poblaciones de ganado criollo comercial y encontraron promedios de $28.92 \pm 3.2 \mathrm{Kg}$ en el departamento de Arauca (17) y en ganado criollo Costeño con cuernos de $29 \pm 3.71 \mathrm{~kg}$ (18). Esto resultados están dentro de los rangos encontrados en este estudio ya que para la característica peso al nacimiento existe una menor influencia del ambiente agroecológico, nutricional y de manejo de las diferentes fincas.

La media hallada para PA270 fue de $216.71 \pm 26.97 \mathrm{~kg}$, fue un promedio normal para cebuínos comerciales pesados al destete y es superada por las reportadas por autores colombianos cuyos valores obtenidos fueron de $241 \pm 30$ $\mathrm{kg}$ y $237.08 \pm 35.60 \mathrm{~kg}$, respectivamente $(3,14)$. Valores similares de $210 \mathrm{~kg}$ para PA270 fueron encontrados para la raza Brahman (19); demostrando así, una variabilidad en el peso de la misma raza por selección y tipos de manejo en cada hato, pudiendo alcanzar pesos entre los 210 y $250 \mathrm{~kg}$ bajo condiciones tropicales colombianas. También existen trabajos que reportan pesos al destete en ganado criollo colombiano en el trópico que oscilan entre $169 \pm 24.7 \mathrm{~kg}$ y $137.65 \pm 28.04 \mathrm{~kg}$, respectivamente $(18,20)$. En Brasil, se obtuvieron pesos para la raza Nelore de $185 \pm 29.40 \mathrm{~kg}$ (15) y en Brahman de México promedios de $180.7 \pm 32.6 \mathrm{~kg}$ (16); estos resultados son diferentes a los del presente estudio, seguramente por las condiciones zootécnicas y ambientales diferentes bajo las cuales fueron evaluadas estas poblaciones de Brasil y México. Al comparar estos resultados se puede inferir que hay gran variabilidad genética y fenotípica entre poblaciones de ganado cebú en diferentes países y que existe un rango de peso que se puede trabajar por selección para mejorar los pesos al destete de la población evaluada en el presente estudio.

La heredabilidad directa y materna, encontrada en este estudio para peso al nacimiento fue baja (0.23) y casi inexistente (0.05), respectivamente. En estudios similares se reportan valores bajos en Brahman comercial de 0.33 y $0.08 ; 0.32$ y 0.16 , respectivamente $(14,21)$ y en ganado Romosinuano de 0.25 y 0.06 , respectivamente (20). Otros autores en ganado Romosinuano criado en condiciones similares a las del presente estudio, encontraron valores por debajo de 0.17 y $0.12(4,22)$. En Brasil en raza Nelore reportan valores de 0.25 y 0.25 , respectivamente $(15,23)$. Las heredabilidades maternas para peso al nacimiento en la mayoría los trabajos fueron muy bajas, lo que significa que la influencia de la genética de la madre es muy poca durante la gestación y la genética aditiva directa y el ambiente uterino tienen un efecto mayor sobre esta característica. La heredabilidad directa fue baja por lo que se debe poner especial atención en mejorar la alimentación y el manejo en el centro de producción evaluado; además, se debe aumentar la presión de selección en las hembras para de esta forma aumentar el desempeño productivo de los terneros y de las hembras madres de las próximas generaciones.

Para PA270 se obtuvo una $\mathrm{h}^{2} \mathrm{~d}$ de $0.39 \pm 0.13$ y $\mathrm{h}^{2} \mathrm{~m}$ de $0.39 \pm 0.091$, estos valores son medios. Los valores encontrados de $h^{2} d$ para PA270 en el presente trabajo están por encima de los reportados en Brahman, Nelore y Romosinuano de $0.22,0.28$ y 0.34 , respectivamente $(4,21,23)$. De esto se puede inferir que la raza Brahman puede obtener individuos con mejor desempeño en su progenie si se realiza un buen proceso de selección genética y apareamiento direccionado. Otros autores en Brahman comercial encentraron valores similares (0.37) de $h^{2} d(14)$ y superiores $(0.45$ y 0.63$)$ en ganado Brangus y Blanco Orejinegro $(7,24)$. Los resultados obtenidos son importantes ya que una heredabilidad media tanto directa como materna, provee la oportunidad para realizar selección y mejoramiento de las características 
productivas tanto en los terneros como en las madres y de esta forma aumentar la eficiencia productiva y reproductiva de los hatos bovinos comerciales. La raza Brahman posee gran potencial por su adaptación al trópico, pero los centros de producción de bovinos de carne comercial no realizan evaluación genética de toros y vacas y por lo tanto no tienen criterio alguno para la selección de los futuros reproductores. Los sistemas productivos que vienen haciendo selección sobre animales con gran potencial genético para la producción de carne tienden a tener mayor productividad de kilogramos/hectárea.

Las correlaciones genéticas ( $\Upsilon \mathrm{g}$ ) y fenotípicas ( $\Upsilon \mathrm{p}$ ) para PN y PA270 fueron bajos posiblemente porque en la población de ganado evaluada no se tenían criterios de selección; además, la característica peso al nacimiento tiende a ser menos influenciada por el ambiente agroecológico, nutricional y de manejo y el peso al destete tiene una mayor influencia del ambiente materno y nutricional; esto hace que las dos características así sean de peso no se correlacionen en gran proporción. Sin embargo, en Colombia otros autores reportan en la misma raza valores de correlación genética de 0.64 , 0.51 y $-0.2(14,19,21)$ y en Brasil en la raza Nelore valores de 0.36 y $0.27(15,23)$. La diferencia entre los valores reportados con los encontrados, se debe al trabajo de selección de animales mejorantes para las dos características que se hace en otras ganaderías especializadas. Las correlaciones fenotípicas encontradas son similares en razas criollas y a las reportadas en otros trabajos realizados en Brahman comercial, con valores de $0.13,0.14$ y 0.16 , respectivamente $(14,18,22)$.

El progreso genético por año para PN y PA270 fue de $-2.02 \mathrm{~kg}$ y $7.81 \mathrm{~kg}$, respectivamente. En la figura $1 \mathrm{se}$ puede observar la ganancia genética del PA270 durante los años 2002 a 2014. En esta figura se nota un aumento en el progreso genético entre los años 2010 a 2014, debido principalmente a la inclusión de programas de inseminación artificial a tiempo fijo, lo que obliga al productor a usar toros evaluados, probados y que poseen gran potencial genético para las características de peso.
En conclusión, las estimaciones de heredabilidad indican la existencia de variabilidad genética aditiva directa, evidenciando la oportunidad de ganancia genética por medio de selección. Sin embargo, las características de PN y PA270 presentan también gran influencia del ambiente, por lo cual se debe poner especial atención al manejo zootécnico y la nutrición de las hembras durante la gestación y de los terneros del nacimiento hasta el destete.

La inseminación artificial es un óptimo medio de introducción de material genéticamente superior en los hatos ganaderos, contribuyendo así con la ganancia genética en las futuras generaciones.

Es importante usar los resultados de la evaluación genética y de la valoración de los animales como estrategia para los apareamientos futuros que permitan un mejoramiento genético de las características de peso en la población de Brahman comercial.

La implementación de programas de mejora genética y de evaluaciones genéticas en ganaderías comerciales puede hacer más eficiente la selección de animales con gran potencial genético, los cuales deben ser incluidos en programas de apareamientos dirigidos; mejorando así, los parámetros productivos de generación en generación y haciendo más rentable la ganadería de carne en Colombia.

\section{Conflicto de intereses}

Los Autores declaramos expresamente a la revista MVZ de la Universidad de Córdoba Colombia, que el manuscrito remitido no ha sido publicado previamente; y que no tenemos conflicto de intereses de ninguna índole.

\section{Agradecimientos}

Agradecemos a la hacienda La Envidia por la disponibilidad de los registros y al grupo de investigación en agronomía y Zootecnia-GIAZ de la Universidad Católica de Oriente, por el acompañamiento durante la investigación.

\section{REFERENCIAS}

1. Organización de las Naciones Unidas para la Alimentación y la Agricultura-FAO. Perspectivas alimentarias (Resumen de mercado). FAO [en línea] 2018 [fecha de acceso 26 de octubre de 2018]; 12. URL disponible en: http://www.fao.org/giews/ reports/food-outlook/es/

2. DANE. La estructura de la producción de carne bovina en Colombia. Sipsa [en línea]. 2012 [fecha de acceso 26 de octubre de 2018]; 4:75. URL disponible en: https://www.dane.gov.co/files/ investigaciones/agropecuario/sipsa/insumos factores de produccion octubre 2012.pdf
3. Montes $D$, Vergara $O$, Prieto $E$, Barragán W. Estimación de la repetibilidad y factores que afectan el peso al nacer y al destete en ganado bovino cebú brahmán. Rev Colombiana Cienc Anim. 2009; 1(1):19-36. https://doi.org/10.24188/recia. v1.n1.2009.405

4. Martínez R y Pérez E. Parámetros y tendencias genéticas para características de crecimiento en el ganado criollo colombiano Romosinuano. Corpoica Cienc Tecnol Agropecu. 2006; 7(1):25-32. https:// doi.org/10.21930/rcta.vol7_num1_art:56

5. Osorio M, Segura C. Factores que afectan el peso al nacer y al destete de becerros de doble propósito en el trópico. Livest Res Rural Dev. 2008; 20(1): Article15. URL Disponible en: http://www. Irrd.org/Irrd20/1/osor20015.htm 
6. Quintero J, Triana J, Quijano J, Arboleda E. Influencia de la inclusión del efecto materno en la estimación de parámetros genéticos del peso al destete en un hato de ganado de carne. Rev Col Cienc Pec. 2007; 20(2):117-123. https://aprendeenlinea.udea.edu. co/revistas/index.php/rccp/article/view/324128

7. Cañas J, Ramírez J, Arboleda O, Ochoa J, Vergara O, Cerón M. Estimación de parámetros genéticos para peso al destete en ganado Blanco Orejinegro (BON) en el noroccidente colombiano. Rev MVZ Córdoba. 2008; 13(1):1138-1145. https://doi.org/10.21897/ $\underline{\mathrm{rmVz} .405}$

8. Castro D. Ensayo sobre tipología de suelos colombianos-Énfasis en génesis y aspectos ambientales. Rev Acad Colomb Cienc. 2003; 27(104):319-341. http://www.accefyn.com/ revista/Vol 27/104/319-341.pdf

9. Kinghorn BP. An algorithm for efficient constrained mate selection. Genet Sel Evol. 2011; 43(1):4. DOI: https://doi.org/10.1186/1297-9686-43-4

10. R Development Core Team. R: a language and environment for statistical computing. [En linea] Version 3.5.1. Vienna: R Foundation for Statistical Computing. 2018. [fecha de acceso 26 de octubre de 2018]; URL disponible en: http://www. Rproject.org/

11. Falconer D, Mackay T. Introduction to Quantitative Genetics. Harlow, UK: Longman Group Ltd; 1996.

12. Boldman $K$, Kriese $L$, Van Vleck $L$, Van Tessell $C$, Kachman S. A manual for use of MTDFREML. A set of programs to obtain estimates of variances and covariances (Draft). USA; USDA-Agric Res Serv: 1995.

13. Daniel Gianola KH. Advances in Statistical Methods for Genetic Improvement of Livestock. vol 18. Lexington, KY: Springer; 1990.

14. Montes V, Vergara G, Prieto M, \& Rodríguez P. Estimación de los parámetros genéticos para el peso al nacer y al destete en ganado bovino de la raza Brahman. Rev MVZ Córdoba. 2008; 13(1):11841191. https://doi.org/10.21897/rmvz.409

15. Chud T, Caetano S, Buzanskas M, Grossi D, Guidolin, D, Nascimento G, Munari D. Genetic analysis for gestation length, birth weight, weaning weight, and accumulated productivity in Nellore beef cattle. Livest Sci. 2014; 170:16-21. https://doi. org/10.1016/j.livsci.2014.09.024

16. Parra G, Martínez J, Cienfuegos E, García F, Ortega E. Parámetros genéticos de variables de crecimiento de ganado Brahman de registro en México. Veterinaria México. 2007; 38(2):217-229. https:// doi.org/10.4067/s0301-732×2009000200004
17. Salamanca C, Quintero V, Benitez M. Características de crecimiento predestete en becerros del sistema doble propósito en el municipio de Arauca. Zoot Trop. $2011 ; 29(4): 455-465$. Disponible en http://www.scielo.org.ve/scielo.php?script=sci arttext\&pid=S0798-72692011000400007\&lng=e s\&nrm =iso.

18. García E, Mitat A, Saraz A, Simanca C, Garay D. Componentes de covarianza y parámetros genéticos para características de crecimiento en una población de ganado Costeño con Cuernos en Colombia. Livest Res Rural Dev. 2015; 27(1):Article6. URL Disponible en: http://www.Irrd.org/Irrd27/1/pere27006.htm

19. Plasse D, Verde O, Fossi H, Romero R, Hoogesteijn R, Bastidas P, Bastardo J. (Co) variance components, genetic parameters and annual trends for calf weights in a pedigree Brahman herd under selection for three decades. J Anim Breed Genet. 2002; 119(3):141-153. https://doi.org/10.1046/j.14390388.2002.00321.x

20. Ossa G, Pérez J, Suárez M. Valores genéticos de caracteres productivos y reproductivos en bovinos Romosinuano. Corpoica Cienc Tecnol Agropecu. 2008; 9(1):93-101. https://doi.org/10.21930/rcta. vol14 num2 art:503

21. Jiménez $A$, Manrique $C$, Martínez $C$. Parámetros y valores genéticos para características de composición corporal, área de ojo de lomo y grasa dorsal medidos mediante ultrasonido en la raza Brahman. Rev Med Vet Zoot 2010; 57(3):159-167. https://revistas.unal.edu.co/index.php/remevez/ article/view/18236

22. Ossa G, Suárez M, Pérez E. Efecto del medio y la herencia sobre el peso al nacimiento, destete y a los 16 meses de edad en terneros de la raza Romosinuano. Corpoica Cienc Tecnol Agropecu. 2007; 8(2):81-92. https://doi.org/10.21930/rcta. vol8 num2 art:98

23. Boligon A, Albuquerque G, Mercadante E, Lôbo B. Herdabilidades e correlações entre pesos do nascimento à idade adulta emrebanhos da raça Nelore. R Bras Zootec. 2009; 38(12):23202326. https://doi.org/10.1590/s151635982009001200005

24. Vergara O, Martínez N, Almanza R, Patiño R, Calderón $A$. Parámetros y tendencias genéticas para característica de crecimiento predestete en una población bovina multirracial en Colombia. Rev Facult Cienc Vet, UCV. 2014; 55(2):68-77. Disponible en http://www.scielo.org.ve/scielo.php?script=sci arttext\&pid=S0258-65762014000200002\&Ing=es \&nrm=iso. 